\title{
How to make the short baseline sterile neutrino compatible with cosmology
}

\author{
Rasmus S. L. Hansen ${ }^{* a}$, Maria Archidiacono ${ }^{b}$, Steen Hannestad ${ }^{c}$ and Thomas $\operatorname{Tram}^{d}$ \\ ${ }^{a}$ Max-Planck-Institut für Kernphysik \\ Saupfercheckweg 1, 69118 Heidelberg, Germany \\ ${ }^{b}$ Institute for Theoretical Particle Physics and Cosmology (TTK) \\ RWTH Aachen University, 52056 Aachen, Germany \\ ${ }^{c}$ Department of Physics and Astronomy, Aarhus University \\ 8000 Aarhus C, Denmark \\ ${ }^{d}$ Institute of Gravitation and Cosmology, University of Portsmouth \\ Dennis Sciama Building, Burnaby Road, Portsmouth, POI 3FX, United Kingdom \\ Email: rasmus@mpi-hd.mpg.de, archidiacono@physik.rwth-aachen.de, \\ steen@aias.au.dk, thomas.tram@port.ac.uk
}

\begin{abstract}
A number of short baseline neutrino experiments have found anomalous results throughout the past decades. These can be interpreted as oscillations between the active neutrinos and a sterile neutrino with a mass around $1 \mathrm{eV}$. However, in the early Universe such a sterile neutrino would be thermalised through its oscillations with Standard Model neutrinos. A fully thermalised sterile neutrino is incompatible with observations of the cosmological microwave background (CMB), large scale structures (LSS) and primordial abundances of the light elements leading to a tension between cosmology and the short baseline neutrino. A new hidden interaction for the sterile neutrino mediated by a vector boson is capable of suppressing oscillations in the Early Universe and delay the production of sterile neutrinos until the Standard Model neutrinos have decoupled. This removes the tension with the light element abundances, but bounds on the neutrino mass from LSS and CMB observations still give tension with the model. Contrarily, a model with a pseudoscalar mediator coupled to the fourth neutrino mass state might relieve all the tension.
\end{abstract}

PACS: 14.60.St, 14.60.Pq, 98.80.Es, 98.80.Cq

38th International Conference on High Energy Physics

3-10 August 2016

Chicago, USA

${ }^{*}$ Speaker. 


\section{Introduction}

Throughout the past two decades, a few anomalies related to short baseline neutrino experiments have arised and still persist [1].

The hypothesis of a sterile neutrino, $v_{s}$, can be constrained in several different ways. If it mixes with $v_{\mu}$ as the LSND anomaly requires [2], $v_{\mu}$ disappearance should also be seen in long baseline experiments. The lack of such $v_{\mu}$ disappearance puts strong limits on the mass and mixing parameters of the sterile neutrino [3]. A similar disappearance of $v_{\mu}$ should also be seen for atmospheric neutrinos in ICECUBE [4]. Although the $v_{\mu}$ disappearance experiments are powerful in constraining the LSND anomaly, $\bar{v}_{e}$ disappearance must be constrained in order to directly address the other anomalies (e.g. $[3,5])$. Some of these experiments are already running, while others will start taking data within the next few years. The current picture when combining all the data is that the LSND/MiniBooNE best fit point is excluded, but parts of the viable parameter space still remain unprobed. The parameters that are still allowed require a mass squared difference $\Delta m_{41}^{2} \sim 1.5 \mathrm{eV}^{2}$ and mixing $\sin ^{2} 2 \theta \sim 0.05[1,6]$.

A sterile neutrino can also be produced in the early Universe and contribute to the energy density. In this proceeding, we will discuss some of the issues regarding its production and constraints that arise from cosmology.

\section{Cosmological bounds}

In the early Universe, the neutrinos were in equilibrium with electrons until $T \sim 1 \mathrm{MeV}$. As a result, the neutrinos are mostly decoupled at electron-positron annihilation, and the neutrino temperature is lower than the photon temperature. The effective number of relativistic species is therefore defined as

$$
N_{\text {eff }}=\frac{\rho_{\text {rel }}}{\rho_{v_{0}}}=\frac{8}{7}\left(\frac{11}{4}\right)^{3 / 4} \frac{\rho_{\text {rel }}}{\rho_{\gamma}}
$$

where $\rho_{v_{0}}$ assumes no heating from electron-positron annihilation. Including heating and QED effects, the Standard Model value of $N_{\text {eff }}$ is 3.045 [7].

It is often assumed that $v_{s}$ is not produced at high temperature and that $v_{s}$ is only produced in the oscillations with active neutrinos, $v_{a}$. The condition for efficient production is that the production rate is larger than the Hubble expansion rate, and this affects $N_{\text {eff }}$ if it occurs before $v_{a}$ decouples. For the parameters considered here, production of sterile neutrinos is dominated by an interplay between oscillations and collisions, where the oscillation rate is larger than the collision rate, $\Gamma_{\text {osc }} \gg \Gamma_{\text {coll }}$. The oscillations average out between each collision, such that the neutrino is in the sterile state with a probability $\frac{1}{2} \sin ^{2}(2 \theta)$. The thermalisation rate is therefore $\Gamma_{\text {therm }}=\frac{1}{2} \sin ^{2}(2 \theta) \Gamma_{\text {coll }}$. Taking the matter background into account, the effective mixing angle is

$$
\sin ^{2}\left(2 \theta_{\text {eff }}\right)=\frac{\sin ^{2}(2 \theta)}{\left(2 E V_{\text {eff }} / \Delta m_{41}^{2}-\cos ^{2} 2 \theta\right)^{2}+\sin ^{2} 2 \theta},
$$

where $V_{\text {eff }}$ is the potential from forward scattering on the background. Calculating $\Gamma / H$ at the temperature where it is maximal gives $\Gamma / H \sim 10^{3} \sin ^{2} 2 \theta\left(\Delta m_{41}^{2} / \mathrm{eV}^{2}\right)^{1 / 2}$. For the relevant mixing parameters $\Gamma / H \gg 1$ at $T>1 \mathrm{MeV}$ resulting in $\Delta N_{\text {eff }}=N_{\text {eff }}-3=1$. 


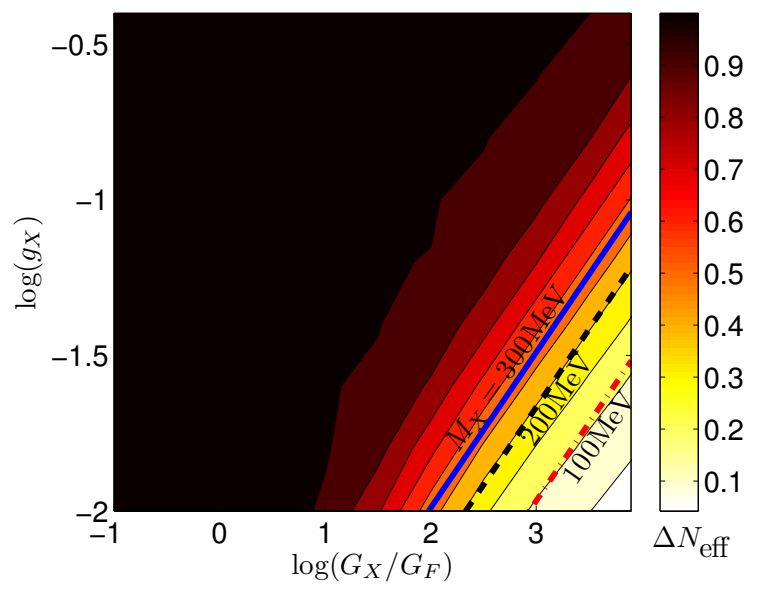

Figure 1: Interactions suppress $v_{s}$ production. Degree of thermalisation depending on the two coupling constants $G_{X}$ and $g_{X}$. The three lines indicate boson masses of 100, 200 and 300MeV. From [10].

A value of $\Delta N_{\text {eff }}=1$ is in tension with the bound from Planck which give $N_{\text {eff }}<3.7$ at 95\% confidence [8] when combining measurements of the cosmological microwave background anisotropies, lensing and measurements of baryon acoustic oscillations (BAO). Additionally, the bound from Big Bang nucleosynthesis is $N_{\text {eff }}<3.2$ at $2 \sigma$ confidence [9]. Apart from the bounds on $N_{\text {eff }}, v_{s}$ would also be dominating $\sum m_{v}$ because of its mass. Since $v_{s}$ could be partially thermalised, the effective mass can be defined as $m_{s}^{\text {eff }}=\left(N_{v_{s}}\right)^{3 / 4} m_{s}$ where $N_{v_{s}}=\rho_{v_{s}} / \rho_{v_{0}}$. In this parameterisation, the combined Planck bounds are $m_{s}^{\text {eff }}<0.52 \mathrm{eV}$ at $95 \%$ confidence [8] when data on BAO are included. This is also in tension with $m_{s} \sim 1 \mathrm{eV}$ and full thermalisation.

\section{Secret interactions}

One way to suppress the production of sterile neutrinos is to suppress the effective mixing angle. This can be done by introducing a large background potential as Equation (2.2) shows. A secret interaction that is only felt by the sterile neutrinos can achieve this [10,11]. In the case of a Fermi-like interaction with the coupling $G_{X}=g_{X}^{2} / M_{X}^{2}$, where $g_{X}$ is the dimensionless coupling constant and $M_{X}$ is the mediator mass, the background potential and collision rate as functions of momentum, are

$$
V_{s}(p)=\frac{16 G_{X}}{3 \sqrt{2} M_{X}^{2}} p u_{v_{s}}, \quad \Gamma_{s}(p)=G_{X}^{2} p T_{v_{s}} n_{v_{s}}
$$

where $u_{v_{s}}$ is the energy density and $n_{v_{s}}$ is the number density of sterile neutrinos. Including these in a numerical calculation, it is found that a sufficiently light mediator can indeed suppress $\Delta N_{\text {eff }}$ as Figure 1 shows. As previously mentioned, the thermalisation rate can be estimated as $\Gamma_{\text {therm }}=\frac{1}{2} \sin ^{2}\left(2 \theta_{\text {eff }}\right) \Gamma_{\text {coll }}$. When both $\theta_{\text {eff }}$ and $\Gamma_{\text {coll }}$ are dominated by the secret interaction, the thermalisation rate is $\Gamma_{\text {therm }} \propto \Gamma_{s} V_{s}^{-2}$. This combination only depends on $M_{X}$ which explains the behaviour of $\Delta N_{\text {eff }}$ as a function of $G_{X}$ and $g_{X}$ in Figure 1.

The Fermi-like interaction is only valid, when $T, E \ll M_{X}$. In the opposite limit where $T, E \gg$ 
$M_{X}$, the background potential and collision rate are given by $[11,12]$

$$
V_{s} \sim \frac{g_{X}^{2} T_{v_{s}}^{2}}{E}, \quad \Gamma_{s} \sim \frac{g_{X}^{4}}{M_{X}^{2}} n_{v_{s}} .
$$

As for the high mass region, the thermalisation rate depends only on $M_{X}$ when combining the potential and collision rate from (3.2). The result is that there is both an upper and a lower mass bound on $M_{X}$ in the limit where $V_{s}$ and $\Gamma_{s}$ dominate $V_{\text {eff }}$ and $\Gamma_{\text {coll }}$.

In the intermediate mass range, the region where $\Delta N_{\text {eff }}$ can be suppressed is confined to $M_{X} \sim$ $0.01-10 \mathrm{MeV}$ and $g_{x} \gtrsim 10^{-3}$ [12]. However, the same study also show that even when $\Delta N_{\text {eff }}=$ 0 , the sterile neutrinos are still produced at temperatures below neutrino decoupling. This has implications for the neutrino mass bound [13]. A simple estimate assumes that $N_{\text {eff }}$ is equally distributed between the three active and the sterile neutrino. Then the effective mass is $m_{s}^{\text {eff }} \gtrsim 0.8 \mathrm{eV}$ which is in conflict with the mass bound from Planck and BAO.

\section{Pseudoscalar mediator}

The secret interaction considered in the previous section was introduced for $v_{s}$ and mediated by a vector boson like the weak interaction in the Standard Model. In this section we will instead look at a secret interaction mediated by a very light pseudoscalar, $\phi$, that couples to the fourth mass state, $v_{4}$.

The coupling to $v_{4}$ implies that the active neutrinos are also coupled to the new pseudoscalar, although the coupling is suppressed by the mixing angle. This leads to the bound $g_{\phi} \lesssim 5 \times 10^{-5}$ from supernova cooling [14].

The background potential and collision rate are very similar to the expressions for a very light vector boson in Equation (3.2). A mass $m_{\phi} \ll 1 \mathrm{eV}$ results in a very large collision rate. In a plasma, this divergence is regulated by screening effects, and using the estimate $m_{\text {screen }} \sim T$ gives $\Gamma_{s} \sim$ $\frac{g_{\phi}^{4}}{T^{2}} n_{v_{4}}$ [15]. This is much smaller than the collision rate from weak interactions for the interesting parameters, so the suppression depends only on the size of $V_{s}$. The condition for dominating the Hamiltonian is $V_{s}>\Delta m_{41}^{2} / T$, and at $T=1 \mathrm{MeV}$, the condition gives $g_{\phi}>\sqrt{\Delta m_{41}^{2}} / T \sim 10^{-6}$. A numerical calculation finds that there is significant suppression at $g_{\phi} \gtrsim 3 \times 10^{-6}$ [16] in good agreement with the order of magnitude estimate.

Although $v_{4}$ and $\phi$ recouple at lower temperatures, this does not affect the Standard Model neutrinos as they propagate as mass states which do not mix with $v_{4}$. Therefore additional $v_{s}$ s are not produced in the way they were produced in Section 3.

While the interaction between $v_{4}$ and $\phi$ at lower temperatures does not lead to additional production of $v_{s}$, it changes how $v_{s}$ affects the CMB anisotropies [17]. The interaction prevents $v_{4}$ from becoming free-streaming, and once $T<m_{v_{4}}, v_{4}$ starts to annihilate into $\phi$. The annihilation leads to an increase in the energy density of the $v_{4}-\phi$ plasma and to a temporary change in the equation of state parameter [17]. In Figure 2 these effects have been taken into account when fitting CMB data, and the consequence is that $N_{\text {fluid }} \sim 0.5$ is preferred, and $m_{s}$ can be consistent with the hints from short baseline experiments. The annihilation of $v_{4}$ to $\phi$ also relaxes any bounds from structure formation that takes place at $T \ll 1 \mathrm{eV}$. $^{1}$

\footnotetext{
${ }^{1}$ The lack free-streaming for $v_{4}$ can also be found in models with a vector mediator and might relax the bounds
} 


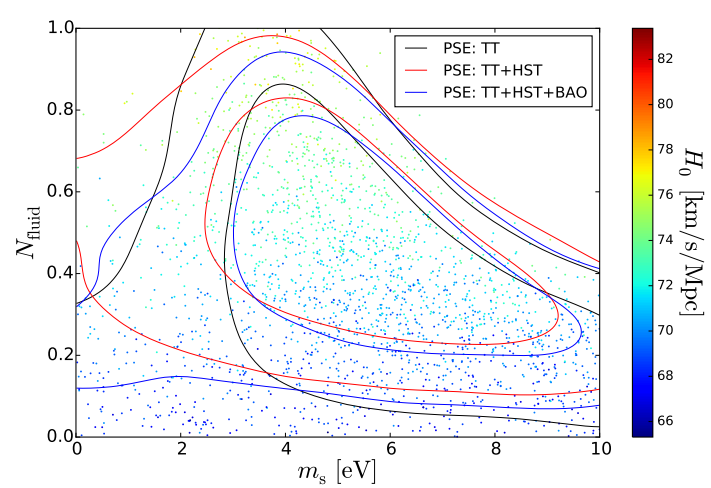

Figure 2: Fit of the pseudoscalar model to cosmological data. 1 and $2 \sigma$ contours when including CMB temperature data from Planck (TT), local measurements of $H_{0}$ (HST) and baryon acoustic oscillations (BAO). The values of three parameter are indicated: The mass of $v_{s}\left(m_{s}\right)$, the combined contribution from $v_{4}$ and $\phi$ to $N_{\text {eff }}\left(N_{\text {fluid }}\right)$ and the Hubble constant $\left(H_{0}\right.$ in the colour scale). From [15].

\section{Conclusions}

The sterile neutrino interpretation of the short baseline anomalies is quite constrained by various experiments, although the final verdict will have to wait until the results from the next generation of dedicated experiments. When the sterile neutrino is introduced to cosmology, it contributes too much to $N_{\text {eff }}$ and with a too large effective mass $m_{s}^{\text {eff }}$.

The large contribution to $N_{\text {eff }}$ can be avoided by introducing a secret interaction that is only felt by $v_{s}$. In this model, the mediator can have a range of masses from $10 \mathrm{keV}$ to $10 \mathrm{MeV}$, but the production of $v_{s}$ might only prevented until $v_{a}$ decouples, and there could be a significant production at lower temperatures. If this is the case, the bound on neutrino masses still excludes almost all of the parameter space.

A model with a light pseudoscalar coupled to $v_{4}$ might circumvent the mass bound because (i) production after decoupling of $v_{a}$ is not possible, (ii) $v_{4}$ and $\phi$ form a tightly coupled fluid which prevents free-streaming from affecting $\mathrm{CMB}$, and (iii) $v_{4}$ annihilates to the much lighter pseudoscalar and therefore leaves no significant imprint on large scale structure formation.

\section{Acknowledgments}

RSLH acknowledges support from the Alexander von Humboldt foundation.

\section{References}

[1] S. Gariazzo, C. Giunti, M. Laveder, Y. F. Li and E. M. Zavanin, Light sterile neutrinos, J. Phys. G 43 (2016) 033001 [arXiv:1507.08204].

[2] A. Aguilar-Arevalo et al. [LSND Collaboration], Evidence for neutrino oscillations from the observation of anti-neutrino(electron) appearance in a anti-neutrino(muon) beam, Phys. Rev. D 64 (2001) 112007 [arXiv:hep-ex/0104049].

from CMB. However, such a model might still be in conflict with observations of structure formation [18]. 
[3] P. Adamson et al. [Daya Bay and MINOS Collaborations], Limits on Active to Sterile Neutrino Oscillations from Disappearance Searches in the MINOS, Daya Bay, and Bugey-3 Experiments, [arXiv:1607.01177].

[4] M. G. Aartsen et al. [IceCube Collaboration], Searches for Sterile Neutrinos with the IceCube Detector, Phys. Rev. Lett. 117 (2016) no.7, 071801 [arXiv:1605.01990].

[5] J. Haser [Stereo Collaboration], Search for eV sterile neutrinos at a nuclear reactor - the Stereo project, J. Phys. Conf. Ser. 718 (2016) no.6, 062023.

[6] G. H. Collin, C. A. Argüelles, J. M. Conrad and M. H. Shaevitz, First Constraints on the Complete Neutrino Mixing Matrix with a Sterile Neutrino, [arXiv:1607.00011].

[7] P. F. de Salas and S. Pastor, Relic neutrino decoupling with flavour oscillations revisited, JCAP 1607 (2016) no.07, 051 [arXiv:1606.06986].

[8] P. A. R. Ade et al. [Planck Collaboration], Planck 2015 results. XIII. Cosmological parameters, Astron. Astrophys. 594 (2016) A13 [arXiv:1502.01589].

[9] R. H. Cyburt, B. D. Fields, K. A. Olive and T. H. Yeh, Big Bang Nucleosynthesis: 2015, Rev. Mod. Phys. 88 (2016) 015004 [arXiv:1505.01076].

[10] S. Hannestad, R. S. Hansen and T. Tram, How Self-Interactions can Reconcile Sterile Neutrinos with Cosmology, Phys. Rev. Lett. 112 (2014) no.3, 031802 [arXiv:1310.5926].

[11] B. Dasgupta and J. Kopp, Cosmologically Safe eV-Scale Sterile Neutrinos and Improved Dark Matter Structure, Phys. Rev. Lett. 112 (2014) no.3, 031803 [arXiv:1310.6337].

[12] J. F. Cherry, A. Friedland and I. M. Shoemaker, Short-baseline neutrino oscillations, Planck, and IceCube, arXiv:1605.06506.

[13] A. Mirizzi, G. Mangano, O. Pisanti and N. Saviano, Collisional production of sterile neutrinos via secret interactions and cosmological implications, Phys. Rev. D 91 (2015) no.2, 025019 [arXiv:1410.1385].

[14] Y. Farzan, Bounds on the coupling of the Majoron to light neutrinos from supernova cooling, Phys. Rev. D 67 (2003) 073015 [arXiv:hep-ph/0211375].

[15] M. Archidiacono, S. Gariazzo, C. Giunti, S. Hannestad, R. Hansen, M. Laveder and T. Tram, Pseudoscalar - sterile neutrino interactions: reconciling the cosmos with neutrino oscillations, JCAP 08(2016)067 [arXiv:1606.07673].

[16] M. Archidiacono, S. Hannestad, R. S. Hansen and T. Tram, Cosmology with self-interacting sterile neutrinos and dark matter - A pseudoscalar model, Phys. Rev. D 91 (2015) no.6, 065021 [arXiv:1404.5915].

[17] M. Archidiacono, S. Hannestad, R. S. Hansen and T. Tram, Sterile neutrinos with pseudoscalar self-interactions and cosmology, Phys. Rev. D 93 (2016) no.4, 045004 [arXiv:1508.02504].

[18] X. Chu, B. Dasgupta and J. Kopp, Sterile neutrinos with secret interactions — lasting friendship with cosmology, JCAP 1510 (2015) no.10, 011 [arXiv:1505.02795]. 